\title{
Article \\ Solvability of an Optimization Problem for the Unsteady Plane Flow of a Non-Newtonian Fluid with Memory
}

\author{
Mikhail A. Artemov \\ Department of Applied Mathematics, Informatics and Mechanics, Voronezh State University, \\ 394018 Voronezh, Russia; artemov_m_a@mail.ru
}

check for

updates

Citation: Artemov, M.A. Solvability of an Optimization Problem for the

Unsteady Plane Flow of a

Non-Newtonian Fluid with Memory. Symmetry 2021, 13, 1026. https:// doi.org/10.3390/sym13061026

Academic Editors: Evgenii S.

Baranovskii, Aleksey P. Zhabko and Vyacheslav Provotorov

Received: 25 April 2021

Accepted: 1 June 2021

Published: 7 June 2021

Publisher's Note: MDPI stays neutral with regard to jurisdictional claims in published maps and institutional affiliations.

Copyright: (C) 2021 by the author. Licensee MDPI, Basel, Switzerland. This article is an open access article distributed under the terms and conditions of the Creative Commons Attribution (CC BY) license (https:/ / creativecommons.org/licenses/by/ $4.0 /)$.

\begin{abstract}
This paper deals with an optimization problem for a nonlinear integro-differential system that describes the unsteady plane motion of an incompressible viscoelastic fluid of Jeffreys-Oldroyd type within a fixed bounded region subject to the no-slip boundary condition. Control parameters are included in the initial condition. The objective of control is to match the velocity field at the final time with a prescribed target field. The control model under consideration is interpreted as a continuous evolution system in an infinite-dimensional Hilbert space. The existence of at least one optimal control is proved under inclusion-type constraints for admissible controls.
\end{abstract}

Keywords: optimization problem; integro-differential system; control operator; existence theorem; non-Newtonian fluid; Jeffreys-Oldroyd viscoelastic fluid; plane flow

\section{Introduction}

It is well known that flow control problems play an important role in the research field of fluid mechanics [1-4]. In particular, the study of control and optimization problems for models of non-Newtonian fluids is a very interesting topic because of their extensive applications in technology and industries. Related mathematical questions attract the attention of many fluid dynamics researchers.

Slawig [5], Guerra [6] and Arada [7] investigated distributed control for the generalized stationary Navier-Stokes equations with shear-dependent viscosity. A paper by Abraham et al. [8] studied numerical shape optimization for a non-Newtonian fluid. In [9], the existence of an optimal weak solution in a control model for the unsteady flow of a viscoelastic medium is established, provided that the initial data are small. The paper [10] is devoted to proving approximate-finite dimensional controllability for linearized motion equations (by neglecting the inertial effects) of the Jeffreys fluid within a bounded flow region with the smooth boundary. Using the surface force as a control parameter, Artemov and Baranovskii [11] established the existence of optimal solutions for a model describing steady-state flows of a nonlinear-viscous incompressible fluid within a bounded region. Optimal boundary control for various viscoelastic fluid systems was analyzed in the works [12-14]. Manakova [15] proved the existence of optimal distributed control for solutions of the Dirichlet-Showalter-Sidorov problem for a model of the dynamics of a weakly viscoelastic fluid. Anh and Nguyet investigated [16] an optimal control problem with a quadratic objective functional and the body force as a control parameter for the three-dimensional Navier-Stokes-Voigt equations, which are a suitable regularization of the classical Navier-Stokes equations and describe the motion of a viscoelastic fluid of Kelvin-Voigt type. Ngan and Toi [17] proposed a finite-dimensional feedback control scheme for stabilizing stationary solutions of the Navier-Stokes-Voigt equations with periodic boundary conditions. Using methods of the theory of pseudo-monotone mappings, Baranovskii [18] constructed an optimal weak solution to the model for the steady-state flow of a dilatant fluid through a container with holes. In [19], certain classes of optimal control problems for the stationary Boussinesq equations with variable density are studied. Evcin et al. [20] analyzed the control of the power-law fluid flow and heat transfer in a 
square duct under an external magnetic field applying the flow index and the Hartmann number. Baranovskii [21] investigated a boundary control problem for the non-isothermal flow of a low-concentrated aqueous polymer solution moving within a fixed bounded region of three-dimensional space $\mathbb{R}^{3}$. Dong and Liu [22] proposed a multi-objective topology optimization method for convective heat transfer problems in a microchannel by using the improved Cross model.

The literature survey indicates that interest in flow control problems for non-Newtonian fluids has grown during the last few years. Despite the large number of works in this subject area, the important case of the initial control (when control parameters are included in the initial conditions) has not yet been studied. Most of the theoretical results were obtained for time-independent flows. Keeping this fact in mind, in this paper, we study an optimal initial control problem for the system of equations governing the unsteady flow of an incompressible viscoelastic fluid of Jeffreys-Oldroyd type [23-25] in a cylinder $Q_{T}=\mathcal{O} \times(0, T)$ :

$$
\begin{gathered}
\operatorname{Re}\left(\partial_{t} \vec{y}+y_{1} \partial_{x_{1}} \vec{y}+y_{2} \partial_{x_{2}} \vec{y}\right)-(1-a) \Delta \vec{y}-\operatorname{div} \mathbb{E}+\operatorname{grad} \pi=\overrightarrow{0}, \quad \forall\left(x_{1}, x_{2}, t\right) \in Q_{T}, \\
\operatorname{div} \vec{y}=0, \quad \forall\left(x_{1}, x_{2}, t\right) \in Q_{T}, \\
\mathbb{E}\left(x_{1}, x_{2}, t\right)=\frac{2 a}{\mathrm{Wi}} \int_{0}^{t} \exp \left(\frac{s-t}{\mathrm{Wi}}\right) \mathbb{D}\left(\vec{y}\left(x_{1}, x_{2}, s\right)\right) d s, \quad \forall\left(x_{1}, x_{2}, t\right) \in Q_{T}, \\
\vec{y}=\overrightarrow{0}, \quad \forall\left(x_{1}, x_{2}, t\right) \in \partial \mathcal{O} \times(0, T), \\
\left.\vec{y}\right|_{t=0}=\vec{u} \in U_{\text {ad }}, \\
\text { Minimize }\left\|\left.\vec{y}\right|_{t=T}-\vec{b}\right\|_{L_{2}} \text { subject to (1)-(5), }
\end{gathered}
$$

where $\mathcal{O} \subset \mathbb{R}^{2}$ is the flow region; $\left(x_{1}, x_{2}\right)$ is a point of $\mathcal{O} ; t$ is the time; $T$ denotes the final moment of time; $\partial_{t}, \partial_{x_{1}}$ and $\partial_{x_{2}}$ are the partial derivatives with respect to $t, x_{1}$ and $x_{2} ; \partial \mathcal{O}$ stands for the boundary of the region $\mathcal{O} ; \vec{y}=\left(y_{1}\left(x_{1}, x_{2}, t\right), y_{2}\left(x_{1}, x_{2}, t\right)\right)$ is the velocity field; $\mathbb{E}=\left(\mathbb{E}_{i j}\left(x_{1}, x_{2}, t\right)\right)_{i, j=1}^{2}$ is the "elastic part" of the stress tensor $(\mathbb{E}$ is symmetric-that is, $\left.\mathbb{E}_{12}=\mathbb{E}_{21}\right) ; \pi=\pi\left(x_{1}, x_{2}, t\right)$ is the pressure function, which includes the potential of body forces; the operators "grad", "div" and $\Delta$ denote, respectively, the gradient, the divergence and the Laplacian with respect to the space variables $x_{1}$ and $x_{2}$; $\mathbb{D}(\vec{y})$ is the symmetric part of the velocity gradient- that is, $\mathbb{D}(\vec{y})=\frac{1}{2}\left(\operatorname{grad} \vec{y}+(\operatorname{grad} \vec{y})^{\top}\right)$; $\operatorname{grad} \vec{y}=\left(\partial_{x_{j}} y_{i}\right)_{i, j=1}^{2} ; \operatorname{Re}$ is the Reynolds number $(\operatorname{Re}>0)$; Wi is the Weissenberg number (Wi $>0) ; a$ is the coupling parameter $(0<a<1) ; \vec{u}=\left(u_{1}\left(x_{1}, x_{2}\right), u_{2}\left(x_{1}, x_{2}\right)\right)$ is a control function; $\vec{b}=\left(b_{1}\left(x_{1}, x_{2}\right), b_{2}\left(x_{1}, x_{2}\right)\right)$ is some desired velocity field; and $U_{\text {ad }}$ denotes the set of admissible controls.

Equation (1) is the balance of linear momentum (Newton's law) in the cylinder $Q_{T}$, and (2) represents the conservation of mass equation (the incompressibility condition). The presence of relation (3) in system (1)-(5) means that the memory on the stresses is taken into account. Note that the use of the exponential memory kernel is typical for Jeffreys-Oldroyd viscoelasticity models (see, e.g., [26,27]). The coupled Equations (1)-(3) describe mediums such as polymer solutions, concrete, bitumens and the earth's crust. The degenerate cases $a=0$ and $a=1$ correspond, respectively, to the classical Navier-Stokes equations (Newtonian fluid) and the Maxwell model. More detailed discussions of the physical background of non-Newtonian fluid models with memory can be found in the survey article by Saut [28].

For the sake of simplicity, we prescribe the standard no-slip condition (4) at impermeable solid walls of the vessel $\mathcal{O}$. However, the proposed approach can also be applied for viscoelastic fluid systems with other physically-relevant boundary conditions, such as the Navier slip [29] and the threshold-slip scenario [30].

The main goal of the present paper is to establish the solvability of optimization problem (6). The structure of this paper is as follows. In the next section, we describe some notation, function spaces and lemmas used in this paper. In Section 3, we introduce 
the definition of admissible triplets ("control-velocity-stress") and study their properties, which we will need further on. Section 4 is devoted to a rigorous formulation of problem (6) by using the appropriate cost functional and the velocity control operator. There we also formulate and prove our main result-Theorem 1-on the existence of optimal controls for integro-differential system (1)-(5).

\section{Preliminaries: Notation, Function Spaces and Auxiliary Statements}

For the reader's convenience, mostly standard notation is used.

The symbol $\triangleq$ is used as "define the thing on the left as the thing on the right".

The symbols $\left\{C_{k}\right\}_{k \in \mathbb{N}}$ denote positive constants that depend only on the data of integro-differential system (1)-(5).

Let $X$ and $Y$ be Banach spaces, and let $\mathcal{A}: D \subset X \rightarrow Y$ be an operator. By $\Gamma(\mathcal{A})$ denote the graph of $\mathcal{A}$; that is,

$$
\Gamma(\mathcal{A}) \triangleq\{(v, \mathcal{A}(v)): v \in D\} \subset X \times Y .
$$

The dual space of $X$ is denoted by $X^{*}$. We shall denote the value of a functional $\ell \in X^{*}$ on an element $\phi \in X$ by $\langle\ell, \phi\rangle_{X^{*} \times X}$ (so-called the "bra-ket" notation).

Let $\mathcal{O}$ be a bounded region in $\mathbb{R}^{2}$. By $L_{p}(\mathcal{O}), 1 \leq p<+\infty$, denote the Lebesgue space with the norm $\|\cdot\|_{L_{p}}$. By $W_{q}^{k}(\mathcal{O}), k \in \mathbb{N}, 1 \leq q<+\infty$, denote the Sobolev space with the norm $\|\cdot\|_{W_{q}^{m}}$. More often, we will deal with the corresponding spaces of vector functions, for which we use the notation $L_{p}\left(\mathcal{O}, \mathbb{R}^{n}\right)$ and $W_{q}^{k}\left(\mathcal{O}, \mathbb{R}^{n}\right)$; that is,

$$
\begin{aligned}
L_{p}\left(\mathcal{O}, \mathbb{R}^{n}\right) & \triangleq \underbrace{L_{p}(\mathcal{O}) \times \cdots \times L_{p}(\mathcal{O})}_{n \text { spaces }}, \\
W_{q}^{k}\left(\mathcal{O}, \mathbb{R}^{n}\right) & \triangleq \underbrace{W_{q}^{k}(\mathcal{O}) \times \cdots \times W_{q}^{k}(\mathcal{O})}_{n \text { spaces }} .
\end{aligned}
$$

Definitions and descriptions of properties of these spaces can be found in $[31,32]$.

By parentheses $(0,0)$ denote the scalar product in the space $L_{2}\left(\mathcal{O}, \mathbb{R}^{n}\right)$; that is,

$$
(\vec{f}, \vec{g}) \triangleq \iint_{\mathcal{O}} \vec{f}\left(x_{1}, x_{2}\right) \cdot \vec{g}\left(x_{1}, x_{2}\right) d x_{1} d x_{2}=\sum_{i=1}^{n} \iint_{\mathcal{O}} f_{i}\left(x_{1}, x_{2}\right) g_{i}\left(x_{1}, x_{2}\right) d x_{1} d x_{2},
$$

for any $\vec{f}, \vec{g} \in L_{2}\left(\mathcal{O}, \mathbb{R}^{n}\right)$.

By definition, put

$$
\begin{gathered}
\mathcal{D}((0, T)) \triangleq\left\{\eta \in C^{\infty}(0, T): \operatorname{supp} \eta \subset(0, T)\right\}, \\
\mathcal{D}(\mathcal{O}) \triangleq\left\{\phi \in C^{\infty}(\mathcal{O}): \operatorname{supp} \phi \subset \mathcal{O}\right\} .
\end{gathered}
$$

Let $H_{0}^{1}(\mathcal{O})$ be the closure of the set $\mathcal{D}(\mathcal{O})$ in the Sobolev space $W_{2}^{1}(\mathcal{O})$.

Lemma 1. If $w \in H_{0}^{1}(\mathcal{O})$, then

$$
\|w\|_{L_{4}} \leq \sqrt[4]{2} \sqrt{\|w\|_{L_{2}}\|\operatorname{grad} w\|_{L_{2}}} .
$$

The last inequality is usually called Ladyzhenskaya's inequality (for the proof, see [33], Chapter III, §3). 
Following [34], we introduce three spaces of functions, which will be widely used in the study of the problem under consideration:

$$
\mathcal{V} \triangleq\left\{\vec{\varphi}=\left(\varphi_{1}, \varphi_{2}\right) \in C^{\infty}\left(\mathcal{O}, \mathbb{R}^{2}\right): \operatorname{div} \vec{\varphi}=0 \text { and } \operatorname{supp} \vec{\varphi} \subset \mathcal{O}\right\} ;
$$

$H$ is the closure of the set $\mathcal{V}$ in the Lebesgue space $L_{2}\left(\mathcal{O}, \mathbb{R}^{2}\right)$;

$V$ is the closure of the set $\mathcal{V}$ in the Sobolev space $W_{2}^{1}\left(\mathcal{O}, \mathbb{R}^{2}\right)$.

We define the scalar product and the associated norm in the space $V$ as follows:

$$
(\vec{\phi}, \vec{\psi})_{V} \triangleq(\operatorname{grad} \vec{\phi}, \operatorname{grad} \vec{\psi}), \quad\|\vec{\phi}\|_{V} \triangleq \sqrt{(\vec{\phi}, \vec{\phi})_{V}} .
$$

From Friedrichs's inequality, it follows that the norm $\|\circ\|_{V}$ is equivalent to $\|\circ\|_{W_{2}^{1}}$.

By using the Riesz representation theorem, one may identify $H$ with the dual space, that is, $H^{*} \simeq H$. Thus, we have the chain of inclusions: $V \hookrightarrow H \simeq H^{*} \hookrightarrow V^{*}$, where the symbol $\hookrightarrow$ denotes a continuous dense embedding.

Recall that the embedding $W_{2}^{1}(\mathcal{O}) \hookrightarrow L_{2}(\mathcal{O})$ is compact (see, e.g., [33], Chapter 2, §1). This yields the following statement.

Lemma 2. The embedding $V \hookrightarrow H$ is compact.

By $\mathbb{M}_{\text {sym }}^{n \times n}$ denote the space of symmetric matrices of dimension $n \times n$.

Let $C([0, T] ; X)$ be the space of continuous functions from $[0, T]$ into $X$, and let $L_{q}(0, T ; X)$ be the space of $L_{q}$-integrable functions from $[0, T]$ into $X$.

Finally, let us formulate one auxiliary result needed for what follows.

Lemma 3. Let $X$ and $Y$ be Hilbert spaces such that $Y \hookrightarrow X \simeq X^{*} \hookrightarrow Y^{*}$. Suppose that

$$
\sigma \in L_{2}(0, T ; Y), \quad \sigma^{\prime} \in L_{2}\left(0, T ; Y^{*}\right) .
$$

Then, the function $\sigma$ is almost everywhere equal to a continuous function from $[0, T]$ into $X$, and we have the following equality, which holds in the scalar distribution sense on $(0, T)$ :

$$
\frac{d}{d t}\|\sigma(t)\|_{X}^{2}=2\left\langle\sigma^{\prime}(t), \sigma(t)\right\rangle_{Y^{*} \times Y}
$$

The proof of this lemma is given in [33], Chapter III, §1.4.

\section{Admissible Triplets of Integro-Differential System (1)-(5) and Their Properties}

Assume the following conditions hold:

(i) The flow region $\mathcal{O}$ is bounded in $\mathbb{R}^{2}$ and the boundary $\partial \mathcal{O}$ is of the class $C^{0,1}$;

(ii) The target function $\vec{b}$ belongs to the space $H$;

(iii) The admissible controls set $U_{\text {ad }}$ is convex, closed and bounded in the space $V$.

Example 1. Consider an example of the admissible controls set that satisfies condition (iii):

$$
U_{\mathrm{ad}} \triangleq\left\{\vec{u} \in V: \quad \iint_{\mathcal{O}}\left|\mathbb{D}\left(\vec{u}\left(x_{1}, x_{2}\right)\right)\right|^{2} d x_{1} d x_{2} \leq r^{2}\right\},
$$

where $r$ is a given number.

Let $\vec{u}: \overline{\mathcal{O}} \rightarrow \mathbb{R}^{2}, \vec{y}: \overline{\mathcal{O}} \times[0, T] \rightarrow \mathbb{R}^{2}$, and $\mathbb{E}: \overline{\mathcal{O}} \times[0, T] \rightarrow \mathbb{M}_{\text {sym }}^{2 \times 2}$.

Definition 1. The triplet $(\vec{u}, \vec{y}, \mathbb{E})$ is called an admissible triplet of integro-differential system (1)-(5) if

$$
(\vec{u}, \vec{y}, \mathbb{E}) \in U_{\mathrm{ad}} \times\left[L_{2}(0, T ; V) \cap C([0, T] ; H)\right] \times C\left([0, T] ; L_{2}\left(\mathcal{O}, \mathbb{M}_{\text {sym }}^{2 \times 2}\right)\right),
$$




$$
\left.\vec{y}\right|_{t=0}=\vec{u},\left.\quad \mathbb{E}\right|_{t=0}=\mathbb{O},
$$

and the following equalities

$$
\begin{gathered}
\operatorname{Re} \frac{d}{d t}(\vec{y}, \vec{z})-\operatorname{Re}\left(y_{1} \vec{y}, \partial_{x_{1}} \vec{z}\right)-\operatorname{Re}\left(y_{2} \vec{y}, \partial_{x_{2}} \vec{z}\right)+(1-a)(\operatorname{grad} \vec{y}, \operatorname{grad} \vec{z})+(\mathbb{E}, \mathbb{D}(\vec{z}))=0, \\
\operatorname{Wi} \frac{d}{d t}(\mathbb{E}, \mathbb{F})+(\mathbb{E}, \mathbb{F})=2 a(\mathbb{D}(\vec{y}), \mathbb{F}),
\end{gathered}
$$

hold in the scalar distribution sense on $(0, T)$, for any test functions $\vec{z} \in V$ and $\mathbb{F} \in L_{2}\left(\mathcal{O}, \mathbb{M}_{\text {sym }}^{2 \times 2}\right)$.

The set of all admissible triplets is denoted by $\Xi\left(U_{\mathrm{ad}}\right)$.

Remark 1. The variational formulation (9), (10) is ordinarily derived from the viscoelastic system (1)-(3) by the Green formula and the following identity

$$
\frac{d}{d t}\left(\int_{0}^{t} \mathbb{K}_{\vec{x}, \vec{y}}(t, s) d s\right) \equiv \mathbb{K}_{\vec{x}, \vec{y}}(t, t)+\int_{0}^{t} \partial_{t} \mathbb{K}_{\vec{x}, \vec{y}}(t, s) d s
$$

with

$$
\mathbb{K}_{\vec{x}, \vec{y}}:[0, T] \times[0, T] \rightarrow \mathbb{M}_{\text {sym }}^{2 \times 2}, \quad \mathbb{K}_{\vec{x}, \vec{y}}(t, s) \triangleq \exp \left(\frac{s-t}{\mathrm{Wi}}\right) \mathbb{D}\left(\vec{y}\left(x_{1}, x_{2}, s\right)\right)
$$

In order to prove the solvability of problem (6), we first study some properties of admissible triplets.

Proposition 1. Suppose $\left(\vec{u}_{i}, \vec{y}_{i}, \mathbb{E}_{i}\right) \in \Xi\left(U_{\mathrm{ad}}\right)$, where $i=1,2$; then

$\sup \left\{\frac{\operatorname{Re}}{2}\left\|\vec{y}_{1}(\tau)-\vec{y}_{2}(\tau)\right\|_{L_{2}}^{2}+\frac{\mathrm{Wi}}{4 a}\left\|\mathbb{E}_{1}(\tau)-\mathbb{E}_{2}(\tau)\right\|_{L_{2}}^{2}: \tau \in[0, T]\right\} \leq \Pi\left(\left\|\vec{u}_{1}-\vec{u}_{2}\right\|_{L_{2}}\right)$,

where $\Pi$ is a non-negative continuous function such that $\Pi(0)=0$.

Proof. The proof proceeds in four steps.

Step 1. First we shall show that

$$
\vec{y}_{i}^{\prime} \in L_{2}\left(0, T ; V^{*}\right), \quad i=1,2 .
$$

Let us introduce operators $\mathscr{A}, \mathscr{B}, \mathscr{C}$ :

$$
\begin{array}{ll}
\mathscr{A}: V \rightarrow V^{*}, & \langle\mathscr{A}(\vec{y}), \vec{z}\rangle_{V^{*} \times V} \triangleq(a-1)(\operatorname{grad} \vec{y}, \operatorname{grad} \vec{z}), \\
\mathscr{B}: V \times V \rightarrow V^{*}, & \left\langle\mathscr{B}\left(\vec{y}, \vec{y}_{0}\right), \vec{z}\right\rangle_{V^{*} \times V} \triangleq \operatorname{Re}\left(y_{1} \vec{y}_{0}, \partial_{x_{1}} \vec{z}\right)+\operatorname{Re}\left(y_{2} \vec{y}_{0}, \partial_{x_{2}} \vec{z}\right), \\
\mathscr{C}: L_{2}\left(\mathcal{O}, \mathbb{M}_{\text {sym }}^{2 \times 2}\right) \rightarrow V^{*}, & \langle\mathscr{C}(\mathbb{E}), \vec{z}\rangle_{V^{*} \times V} \triangleq-(\mathbb{E}, \mathbb{D}(\vec{z})) .
\end{array}
$$

From Definition 1 it follows that

$$
\operatorname{Re} \vec{y}_{i}^{\prime}=\mathscr{A}\left(\vec{y}_{i}\right)+\mathscr{B}\left(\vec{y}_{i}, \vec{y}_{i}\right)+\mathscr{C}\left(\mathbb{E}_{i}\right), \quad i=1,2 .
$$

Clearly, we have the following inclusions:

$$
\mathscr{A}\left(\vec{y}_{i}\right) \in L_{2}\left(0, T ; V^{*}\right), \mathscr{C}\left(\mathbb{E}_{i}\right) \in L_{2}\left(0, T ; V^{*}\right), i=1,2 .
$$

Therefore, it remains to check that

$$
\mathscr{B}\left(\vec{y}_{i}, \vec{y}_{i}\right) \in L_{2}\left(0, T ; V^{*}\right), \quad i=1,2 .
$$


Indeed, using Hölder's inequality and Ladyzhenskaya's inequality (7), we obtain

$$
\begin{aligned}
\left|\left\langle\mathscr{B}\left(\vec{y}_{i}(t), \vec{y}_{i}(t)\right), \vec{z}\right\rangle_{V^{*} \times V}\right| & \leq C_{1}\left\|\vec{y}_{i}(t)\right\|_{L_{4}}^{2}\|\vec{z}\|_{V} \\
& \leq C_{2}\left\|\vec{y}_{i}(t)\right\|_{L_{2}}\left\|\operatorname{grad} \vec{y}_{i}(t)\right\|_{L_{2}}\|\vec{z}\|_{V} .
\end{aligned}
$$

From (16) and the inclusions

$$
\vec{y}_{i} \in C\left([0, T], L_{2}\left(\mathcal{O}, \mathbb{R}^{2}\right)\right), \quad i=1,2,
$$

it follows that (15) holds.

Taking into account (13)-(15), we conclude that both inclusions from (12) hold.

Step 2. Let us prove that

$$
\mathbb{E}_{i}^{\prime} \in L_{2}\left(0, T ; L_{2}^{*}\left(\mathcal{O}, \mathbb{M}_{\mathrm{sym}}^{2 \times 2}\right)\right), \quad i=1,2 .
$$

Consider operators $\mathscr{K}$ and $\mathscr{N}$ :

$$
\begin{array}{ll}
\mathscr{K}: L_{2}\left(\mathcal{O}, \mathbb{M}_{\text {sym }}^{2 \times 2}\right) \rightarrow L_{2}^{*}\left(\mathcal{O}, \mathbb{M}_{\text {sym }}^{2 \times 2}\right), & \langle\mathscr{K}(\mathbb{E}), \mathbb{F}\rangle_{L_{2}^{*} \times L_{2}} \triangleq-(\mathbb{E}, \mathbb{F}), \\
\mathscr{N}: V \rightarrow L_{2}^{*}\left(\mathcal{O}, \mathbb{M}_{\text {sym }}^{2 \times 2}\right), & \langle\mathscr{N}(\vec{y}), \mathbb{F}\rangle_{L_{2}^{*} \times L_{2}} \triangleq 2 a(\mathbb{D}(\vec{y}), \mathbb{F}) .
\end{array}
$$

From Definition 1 it follows that

$$
\mathrm{Wi} \mathbb{E}_{i}^{\prime}=\mathscr{K}\left(\mathbb{E}_{i}\right)+\mathscr{N}\left(\vec{y}_{i}\right), \quad i=1,2 .
$$

It is directly verifiable that for the terms on the right-hand side of the last equality, the following inclusions are true:

$$
\mathscr{K}\left(\mathbb{E}_{i}\right) \in L_{2}\left(0, T ; L_{2}^{*}\left(\mathcal{O}, \mathbb{M}_{\text {sym }}^{2 \times 2}\right)\right), \mathscr{N}\left(\vec{y}_{i}\right) \in L_{2}\left(0, T ; L_{2}^{*}\left(\mathcal{O}, \mathbb{M}_{\text {sym }}^{2 \times 2}\right)\right), i=1,2,
$$

whence (17).

Step 3. Let $\vec{w} \triangleq \vec{y}_{1}-\vec{y}_{2}$ and $\mathbb{G} \triangleq \mathbb{E}_{1}-\mathbb{E}_{2}$. Taking into account (12) and (17), we apply Lemma 3 to functions $\vec{w}$ and $\mathbb{G}$; this gives the following two equalities

$$
\frac{d}{d t}\|\vec{w}(t)\|_{L_{2}}^{2}=2\left\langle\vec{w}^{\prime}(t), \vec{w}(t)\right\rangle_{V^{*} \times V}, \quad \frac{d}{d t}\|\mathbb{G}(t)\|_{L_{2}}^{2}=2\left\langle\mathbb{G}^{\prime}(t), \mathbb{G}(t)\right\rangle_{L_{2}^{*} \times L_{2}}
$$

which hold for almost all $t \in(0, T)$.

Step 4. Since $\left(\vec{u}_{i}, \vec{y}_{i}, \mathbb{E}_{i}\right) \in \Xi\left(U_{\mathrm{ad}}\right), i=1,2$, it is not hard to establish that

$$
\begin{gathered}
\operatorname{Re}\left\langle\vec{w}^{\prime}, \vec{z}\right\rangle_{V^{*} \times V}-\operatorname{Re}\left(y_{11} \vec{w}, \partial_{x_{1}} \vec{z}\right)-\operatorname{Re}\left(y_{12} \vec{w}, \partial_{x_{2}} \vec{z}\right)-\operatorname{Re}\left(w_{1} \vec{y}_{2}, \partial_{x_{1}} \vec{z}\right) \\
-\operatorname{Re}\left(w_{2} \vec{y}_{2}, \partial_{x_{2}} \vec{z}\right)+(\mathbb{G}, \mathbb{D}(\vec{z}))+(1-a)(\operatorname{grad} \vec{w}, \operatorname{grad} \vec{z})=0, \\
\operatorname{Wi}\left\langle\mathbb{G}^{\prime}, \mathbb{F}\right\rangle_{L_{2}^{*} \times L_{2}}+(\mathbb{G}, \mathbb{F})=2 a(\mathbb{D}(\vec{w}), \mathbb{F}),
\end{gathered}
$$

for any $\vec{z} \in V$ and $\mathbb{F} \in L_{2}\left(\mathcal{O}, \mathbb{M}_{\text {sym }}^{2 \times 2}\right)$.

Setting

$$
\vec{z}(t) \equiv \vec{w}(t), \quad \mathbb{F}(t) \equiv \frac{1}{2 a} \mathbb{G}(t)
$$

into (19) and (20), respectively, we add the obtained equalities. Using (18) and the following relations

$$
-\operatorname{Re}\left(y_{11} \vec{w}, \partial_{x_{1}} \vec{z}\right)-\operatorname{Re}\left(y_{12} \vec{w}, \partial_{x_{2}} \vec{z}\right)=\left\langle\mathscr{B}\left(-\vec{y}_{1}, \vec{w}\right), \vec{w}\right\rangle_{V^{*} \times V}=0,
$$

we arrive at the equality 


$$
\begin{gathered}
\frac{\operatorname{Re}}{2} \frac{d}{d t}\|\vec{w}(t)\|_{L_{2}}^{2}-\operatorname{Re}\left(w_{1}(t) \vec{y}_{2}(t), \partial_{x_{1}} \vec{w}(t)\right)-\operatorname{Re}\left(w_{2}(t) \vec{y}_{2}(t), \partial_{x_{2}} \vec{w}(t)\right) \\
+(1-a)\|\vec{w}(t)\|_{V}^{2}+\frac{W i}{4 a} \frac{d}{d t}\|\mathbb{G}(t)\|_{L_{2}}^{2}+\frac{1}{2 a}\|\mathbb{G}(t)\|_{L_{2}}^{2}=0 .
\end{gathered}
$$

Let us estimate the sum of the second and third terms on the left-hand side of (21). Using the formula for integration by parts and applying the Hölder, Young, and Ladyzhenskaya inequalities, we obtain

$$
\begin{aligned}
& \left|\operatorname{Re}\left(w_{1}(t) \vec{y}_{2}(t), \partial_{x_{1}} \vec{w}(t)\right)+\operatorname{Re}\left(w_{2}(t) \vec{y}_{2}(t), \partial_{x_{2}} \vec{w}(t)\right)\right| \\
& \quad=\operatorname{Re}\left|\left(w_{1}(t) \partial_{x_{1}} \vec{y}_{2}(t), \vec{w}(t)\right)+\left(w_{2}(t) \partial_{x_{2}} \vec{y}_{2}(t), \vec{w}(t)\right)\right| \\
& \leq C_{3}\|\vec{w}(t)\|_{L_{4}}^{2}\left\|\vec{y}_{2}(t)\right\|_{V} \\
& \leq C_{4}\|\vec{w}(t)\|_{L_{2}}\|\operatorname{grad} \vec{w}(t)\|_{L_{2}}\left\|\vec{y}_{2}(t)\right\|_{V} \\
& =C_{4}\|\vec{w}(t)\|_{L_{2}}\|\vec{w}(t)\|_{V}\left\|\vec{y}_{2}(t)\right\|_{V} \\
& \leq C_{5}\|\vec{w}(t)\|_{L_{2}}^{2}\left\|\vec{y}_{2}(t)\right\|_{V}^{2}+(1-a)\|\vec{w}(t)\|_{V}^{2} .
\end{aligned}
$$

Due to (22), we derive from (21) the following estimate

$$
\frac{d}{d t}\left(\frac{\operatorname{Re}}{2}\|\vec{w}(t)\|_{L_{2}}^{2}+\frac{W i}{4 a}\|\mathbb{G}(t)\|_{L_{2}}^{2}\right) \leq \zeta(t)\left(\frac{\operatorname{Re}}{2}\|\vec{w}(t)\|_{L_{2}}^{2}+\frac{W i}{4 a}\|\mathbb{G}(t)\|_{L_{2}}^{2}\right),
$$

where

$$
\zeta:[0, T] \rightarrow \mathbb{R}, \quad \zeta(t) \triangleq C_{6}\left\|\vec{y}_{2}(t)\right\|_{V}^{2} .
$$

Since $\vec{y}_{2} \in L_{2}(0, T ; V)$, we see that $\zeta \in L_{1}(0, T)$. Therefore, we can apply the Grönwall lemma to (23) and obtain

$$
\frac{\operatorname{Re}}{2}\|\vec{w}(t)\|_{L_{2}}^{2}+\frac{W i}{4 a}\|\mathbb{G}(t)\|_{L_{2}}^{2} \leq\left(\frac{\operatorname{Re}}{2}\|\vec{w}(0)\|_{L_{2}}^{2}+\frac{W i}{4 a}\|\mathbb{G}(0)\|_{L_{2}}^{2}\right) \exp \left(\|\zeta\|_{L_{1}(0, T)}\right),
$$

for any $t \in(0, T)$. Further, using the relations

$$
\begin{aligned}
& \vec{w}(0)=\vec{y}_{1}(0)-\vec{y}_{2}(0)=\vec{u}_{1}-\vec{u}_{2}, \\
& \mathbb{G}(0)=\mathbb{E}_{1}(0)-\mathbb{E}_{2}(0)=\mathbb{O},
\end{aligned}
$$

we arrive at inequality

$$
\frac{\operatorname{Re}}{2}\|\vec{w}(t)\|_{L_{2}}^{2}+\frac{W i}{4 a}\|\mathbb{G}(t)\|_{L_{2}}^{2} \leq \frac{\operatorname{Re}}{2}\left\|\vec{u}_{1}-\vec{u}_{2}\right\|_{L_{2}}^{2} \exp \left(\|\zeta\|_{L_{1}(0, T)}\right), \quad \forall t \in[0, T] .
$$

This yields required inequality (11) with the function $\Pi$ defined as follows:

$$
\Pi:[0,+\infty) \rightarrow[0,+\infty), \quad \Pi(s) \triangleq \frac{\operatorname{Re}}{2} s^{2} \exp \left(C_{7}\right)
$$

with

$$
C_{7} \triangleq C_{6} \sup \left\{\|\vec{y}\|_{L_{2}(0, T ; V)}^{2}:(\vec{u}, \vec{y}, \mathbb{E}) \in \Xi\left(U_{\mathrm{ad}}\right)\right\}<+\infty .
$$

Thus, Proposition 1 is proved.

Corollary 1. Suppose $\left(\vec{u}, \vec{y}_{i}, \mathbb{E}_{i}\right) \in \Xi\left(U_{\mathrm{ad}}\right)$, where $i=1,2$; then

$$
\vec{y}_{1}(t) \equiv \vec{y}_{2}(t), \quad \mathbb{E}_{1}(t) \equiv \mathbb{E}_{2}(t), \quad \forall t \in[0, T] .
$$

Proposition 2. For any vector-valued function $\vec{u}$ from the set $U_{\mathrm{ad}}$, there exists a unique pair $(\vec{y}, \mathbb{E})$ such that $(\vec{u}, \vec{y}, \mathbb{E}) \in \Xi\left(U_{\mathrm{ad}}\right)$. 
Proof. Following the general line of the approach proposed in [29], in order to construct $\vec{y}$ and $\mathbb{E}$, we shall apply the Faedo-Galerkin scheme.

Let $\left\{\vec{z}_{k}\right\}_{k \in \mathbb{N}}$ be a total sequence of vector functions in both the spaces $H$ and $V$ such that this sequence is an orthonormal basis of $H$. To construct such a sequence, one can use a technique that is based on the analysis of solutions to a spectral problem with the symmetry and compactness properties (see, [34], Chapter 1, §6.3).

Moreover, we fix some orthonormal basis $\left\{\mathbb{F}_{k}\right\}_{k \in \mathbb{N}}$ in the space $L_{2}\left(\mathcal{O}, \mathbb{M}_{\text {sym }}^{2 \times 2}\right)$.

Take an arbitrary natural number $n$. The Faedo-Galerkin method reads:

Find $\vec{y}_{n}$ and $\mathbb{E}_{n}$ of the form

$$
\begin{aligned}
& \vec{y}_{n}\left(x_{1}, x_{2}, t\right) \triangleq \sum_{k=1}^{n} a_{n, k}(t) \vec{z}_{k}\left(x_{1}, x_{2}\right), \\
& \mathbb{E}_{n}\left(x_{1}, x_{2}, t\right) \triangleq \sum_{k=1}^{n} B_{n, k}(t) \mathbb{F}_{k}\left(x_{1}, x_{2}\right),
\end{aligned}
$$

where $a_{n, k}:[0, T] \rightarrow \mathbb{R}$ and $B_{n, k}:[0, T] \rightarrow \mathbb{R}$ are unknown functions, satisfying the finitedimensional approximate problem:

$$
\begin{gathered}
\operatorname{Re}\left(\partial_{t} \vec{y}_{n}, \vec{z}_{k}\right)+\operatorname{Re}\left(y_{n 1} \partial_{x_{1}} \vec{y}_{n}, \vec{z}_{k}\right)+\operatorname{Re}\left(y_{n 2} \partial_{x_{2}} \vec{y}_{n}, \vec{z}_{k}\right)+\left(\mathbb{E}_{n}, \mathbb{D}\left(\vec{z}_{k}\right)\right) \\
+(1-a)\left(\operatorname{grad} \vec{y}_{n}, \operatorname{grad} \vec{z}_{k}\right)=0, \quad t \in(0, T), k=1, \ldots, n, \\
\operatorname{Wi}\left(\partial_{t} \mathbb{E}_{n}, \mathbb{F}_{k}\right)+\left(\mathbb{E}_{n}, \mathbb{F}_{k}\right)=2 a\left(\mathbb{D}\left(\vec{y}_{n}\right), \mathbb{F}_{k}\right), \quad t \in(0, T), k=1, \ldots, n, \\
\vec{y}_{n}\left(x_{1}, x_{2}, 0\right)=\sum_{k=1}^{n}\left(\vec{u}, \vec{z}_{k}\right) \vec{z}_{k}\left(x_{1}, x_{2}\right), \quad\left(x_{1}, x_{2}\right) \in \mathcal{O}, \\
\mathbb{E}_{n}\left(x_{1}, x_{2}, 0\right)=\mathbb{O}, \quad\left(x_{1}, x_{2}\right) \in \mathcal{O} .
\end{gathered}
$$

For the Faedo-Galerkin solutions $\left(\vec{y}_{n}, \mathbb{E}_{n}\right)$, we shall derive global-in-time a priori estimates independent of the parameter $n$. Such estimates ensure the solvability of the Cauchy problem (24)-(27) on the entire interval $[0, T]$ for any $n \in \mathbb{N}$.

Suppose the pair $\left(\vec{y}_{n}, \mathbb{E}_{n}\right)$ satisfies system (24)-(27). Multiply (24) by $a_{n, k}(t)$ and sum the resulting equalities for $k=1, \ldots, n$; this gives

$$
\begin{gathered}
\operatorname{Re}\left(\partial_{t} \vec{y}_{n}, \vec{y}_{n}\right)+\operatorname{Re}\left(y_{n 1} \partial_{x_{1}} \vec{y}_{n}, \vec{y}_{n}\right)+\operatorname{Re}\left(y_{n 2} \partial_{x_{2}} \vec{y}_{n}, \vec{y}_{n}\right) \\
+\left(\mathbb{E}_{n}, \mathbb{D}\left(\vec{y}_{n}\right)\right)+(1-a)\left(\operatorname{grad} \vec{y}_{n}, \operatorname{grad} \vec{y}_{n}\right), \quad t \in(0, T) .
\end{gathered}
$$

Since

$$
\operatorname{Re}\left(y_{n 1} \partial_{x_{1}} \vec{y}_{n}, \vec{y}_{n}\right)+\operatorname{Re}\left(y_{n 2} \partial_{x_{2}} \vec{y}_{n}, \vec{y}_{n}\right)=-\left\langle\mathscr{B}\left(\vec{y}_{n}, \vec{y}_{n}\right), \vec{y}_{n}\right\rangle_{V^{*} \times V}=0,
$$

we see that (28) reduces to

$$
\operatorname{Re}\left(\partial_{t} \vec{y}_{n}, \vec{y}_{n}\right)+\left(\mathbb{E}_{n}, \mathbb{D}\left(\vec{y}_{n}\right)\right)+(1-a)\left(\operatorname{grad} \vec{y}_{n}, \operatorname{grad} \vec{y}_{n}\right)=0, \quad t \in(0, T) .
$$

Further, we multiply (25) by $B_{n, k}(t)$ and sum up the resulting equalities for $k=1, \ldots, n$. This yields the following equality:

$$
\operatorname{Wi}\left(\partial_{t} \mathbb{E}_{n}, \mathbb{E}_{n}\right)+\left(\mathbb{E}_{n}, \mathbb{E}_{n}\right)=2 a\left(\mathbb{D}\left(\vec{y}_{n}\right), \mathbb{E}_{n}\right), \quad t \in(0, T) .
$$

Next, we multiply (29) by $2 a$ and sum the result with (30); this gives

$$
2 a \operatorname{Re}\left(\partial_{t} \vec{y}_{n}, \vec{y}_{n}\right)+2 a(1-a)\left(\operatorname{grad} \vec{y}_{n}, \operatorname{grad} \vec{y}_{n}\right)+\left(\mathbb{E}_{n}, \mathbb{E}_{n}\right)+\mathrm{Wi}\left(\partial_{t} \mathbb{E}_{n}, \mathbb{E}_{n}\right)=0, \quad t \in(0, T) .
$$

Whence

$$
a \operatorname{Re} \frac{d}{d t}\left\|\vec{y}_{n}\right\|_{L_{2}}^{2}+2 a(1-a)\left\|\operatorname{grad} \vec{y}_{n}\right\|_{L_{2}}^{2}+\left\|\mathbb{E}_{n}\right\|_{L_{2}}^{2}+\frac{W i}{2} \frac{d}{d t}\left\|\mathbb{E}_{n}\right\|_{L_{2}}^{2}=0, \quad t \in(0, T) .
$$


By integrating the last equality with respect to $t$, from 0 to $\tau$, we find that

$$
\begin{gathered}
a \operatorname{Re}\left\|\vec{y}_{n}(\tau)\right\|_{L_{2}}^{2}+2 a(1-a) \int_{0}^{\tau}\left\|\operatorname{grad} \vec{y}_{n}(t)\right\|_{L_{2}}^{2} d t+\int_{0}^{\tau}\left\|\mathbb{E}_{n}(t)\right\|_{L_{2}}^{2} d t \\
+\frac{W \mathrm{i}}{2}\left\|\mathbb{E}_{n}(\tau)\right\|_{L_{2}}^{2}=a \operatorname{Re}\left\|\vec{y}_{n}(0)\right\|_{L_{2}}^{2}, \quad \tau \in[0, T] .
\end{gathered}
$$

Taking into account the relations

$$
\begin{gathered}
\left\|\vec{y}_{n}(0)\right\|_{L_{2}} \leq\|\vec{u}\|_{L_{2}} \leq \sup \left\{\|\vec{h}\|_{L_{2}}: \vec{h} \in U_{\mathrm{ad}}\right\}=C_{8}<+\infty, \\
\left\|\operatorname{grad} \vec{y}_{n}(t)\right\|_{L_{2}}=\left\|\vec{y}_{n}(t)\right\|_{V}, \quad t \in(0, T),
\end{gathered}
$$

we deduce from (31) the following estimate:

$$
\begin{gathered}
a \operatorname{Re}\left\|\vec{y}_{n}(\tau)\right\|_{L_{2}}^{2}+2 a(1-a) \int_{0}^{\tau}\left\|\vec{y}_{n}(t)\right\|_{V}^{2} d t+\int_{0}^{\tau}\left\|\mathbb{E}_{n}(t)\right\|_{L_{2}}^{2} d t \\
+\frac{\mathrm{Wi}}{2}\left\|\mathbb{E}_{n}(\tau)\right\|_{L_{2}}^{2} \leq a \operatorname{Re} C_{8}^{2}, \quad \tau \in[0, T] .
\end{gathered}
$$

From this estimate and condition (iii), it follows that the

$$
\begin{aligned}
& \sup \left\{\left\|\vec{y}_{n}(\tau)\right\|_{L_{2}}: n \in \mathbb{N}, \tau \in[0, T]\right\}<+\infty, \\
& \sup \left\{\left\|\mathbb{E}_{n}(\tau)\right\|_{L_{2}}: n \in \mathbb{N}, \tau \in[0, T]\right\}<+\infty .
\end{aligned}
$$

This implies that the Cauchy problem (24)-(27) is solvable on the interval $[0, T]$.

In view of inequality (32), we also have

$$
\sup \left\{\left\|\vec{y}_{n}\right\|_{L_{2}(0, T ; V)}: n \in \mathbb{N}\right\}<+\infty,
$$

Moreover, using techniques similar to those employed for constructing solutions to the evolution Navier-Stokes equations (see [34], Chapter 1, §6.4]), one can deduce

$$
\sup \left\{\left\|\vec{y}_{n}^{\prime}\right\|_{L_{2}\left(0, T ; V^{*}\right)}: n \in \mathbb{N}\right\}<+\infty .
$$

Therefore, without loss of generality, we can assume that

$$
\begin{aligned}
& \vec{y}_{n} \text { converges to } \vec{y} \text { weakly in } L_{2}(0, T ; V) \text { as } n \rightarrow+\infty, \\
& \vec{y}_{n} \text { converges to } \vec{y} \text { strongly in } L_{2}(0, T ; H) \text { as } n \rightarrow+\infty, \\
& \mathbb{E}_{n} \text { converges to } \mathbb{E} \text { weakly in } L_{2}\left(0, T ; L_{2}\left(\mathcal{O}, \mathbb{M}_{\text {sym }}^{2 \times 2}\right)\right) \text { as } n \rightarrow+\infty,
\end{aligned}
$$

for some $\vec{y}$ and $\mathbb{E}$.

We multiply (24) by an arbitrary function $\xi \in \mathcal{D}(0, T)$ and integrate with respect to $t$ from 0 to $T$. By integrating by parts the first term in the left-hand side of the obtained equality, we find that

$$
\begin{gathered}
-\operatorname{Re} \int_{0}^{T}\left(\vec{y}_{n}, \vec{z}_{k}\right) \xi^{\prime} d t+\operatorname{Re} \int_{0}^{T}\left(y_{n 1} \partial_{x_{1}} \vec{y}_{n}, \vec{z}_{k}\right) \xi d t+\operatorname{Re} \int_{0}^{T}\left(y_{n 2} \partial_{x_{2}} \vec{y}_{n}, \vec{z}_{k}\right) \xi d t \\
+\int_{0}^{T}\left(\mathbb{E}_{n}, \mathbb{D}\left(\vec{z}_{k}\right)\right) \xi d t+(1-a) \int_{0}^{T}\left(\operatorname{grad} \vec{y}_{n}, \operatorname{grad} \vec{z}_{k}\right) \xi d t=0 .
\end{gathered}
$$

Next, we multiply (25) by function $\xi$ and integrate with respect to $t$ from 0 to $T$. By integrating by parts the first term in the left-hand side of the obtained equality, we get

$$
\text { - Wi } \int_{0}^{T}\left(\mathbb{E}_{n}, \mathbb{F}_{k}\right) \xi^{\prime} d t+\int_{0}^{T}\left(\mathbb{E}_{n}, \mathbb{F}_{k}\right) \xi d t=2 a \int_{0}^{T}\left(\mathbb{D}\left(\vec{y}_{n}\right), \mathbb{F}_{k}\right) \xi d t
$$


Taking into account (33)-(35), we pass to the limit $n \rightarrow+\infty$ in equalities (36) and (37); this gives

$$
\begin{gathered}
-\operatorname{Re} \int_{0}^{T}\left(\vec{y}, \vec{z}_{k}\right) \xi^{\prime} d t+\operatorname{Re} \int_{0}^{T}\left(y_{1} \partial_{x_{1}} \vec{y}, \vec{z}_{k}\right) \xi d t+\operatorname{Re} \int_{0}^{T}\left(y_{2} \partial_{x_{2}} \vec{y}_{,} \vec{z}_{k}\right) \xi d t \\
\quad+\int_{0}^{T}\left(\mathbb{E}, \mathbb{D}\left(\vec{z}_{k}\right)\right) \xi d t+(1-a) \int_{0}^{T}\left(\operatorname{grad} \vec{y}, \operatorname{grad} \vec{z}_{k}\right) \xi d t=0, \\
-\mathrm{Wi} \int_{0}^{T}\left(\mathbb{E}, \mathbb{F}_{k}\right) \xi^{\prime} d t+\int_{0}^{T}\left(\mathbb{E}, \mathbb{F}_{k}\right) \xi d t=2 a \int_{0}^{T}\left(\mathbb{D}(\vec{y}), \mathbb{F}_{k}\right) \xi d t,
\end{gathered}
$$

for each $k \in \mathbb{N}$.

Since the sequence $\left\{\vec{z}_{k}\right\}_{k \in \mathbb{N}}$ is total in the space $V$ and the sequence $\left\{\mathbb{F}_{k}\right\}_{k \in \mathbb{N}}$ is total in $L_{2}\left(\mathcal{O}, \mathbb{M}_{\text {sym }}^{2 \times 2}\right)$, we see that equalities (38) and (39) remain valid if we replace $\vec{z}_{k}$ and $\mathbb{F}_{k}$ with arbitrary functions $\vec{z} \in V$ and $\mathbb{F} \in L_{2}\left(\mathcal{O}, \mathbb{M}_{\text {sym }}^{2 \times 2}\right)$, respectively.

Moreover, in view of relations (26) and (27), we arrive at both equalities from (8).

Thus, we have established that $(\vec{u}, \vec{y}, \mathbb{E}) \in \Xi\left(U_{\mathrm{ad}}\right)$.

The uniqueness of the pair $(\vec{y}, \mathbb{E})$ satisfying the conditions of this proposition follows directly from Proposition 1 . The proof is completed.

\section{The Operator Setting of Optimization Problem (6) and the Main Result}

In the previous section, it is shown that, for any choice of control $\vec{u}$ from the set $U_{\mathrm{ad}}$, there exists a unique triplet $(\vec{u}, \vec{y}, \mathbb{E})$ belonging to the set $\Xi\left(U_{\mathrm{ad}}\right)$. This allows us to interpret control model (1)-(5) as a continuous evolution system in the Cartesian product $H \times L_{2}\left(\mathcal{O}, \mathbb{M}_{\text {sym }}^{2 \times 2}\right)$ and correctly define the control operators for both the velocity and stress fields.

Definition 2. The velocity control operator $\Phi_{\mathrm{vel}}$ is a map from $[0, T] \times U_{\mathrm{ad}}$ into $H$ that is defined by the following formula:

$$
\Phi_{\mathrm{vel}}(t, \vec{u}) \triangleq \vec{y}(t),
$$

where the vector-valued function $\vec{y}$ is the second component of the admissible triplet $(\vec{u}, \vec{y}, \mathbb{E})$.

Definition 3. The stress control operator $\Phi_{\text {stress }}$ is a map from $[0, T] \times U_{\text {ad }}$ into $L_{2}\left(\mathcal{O}, \mathbb{M}_{\mathrm{sym}}^{2 \times 2}\right)$ that is defined by the following formula:

$$
\Phi_{\text {stress }}(t, \vec{u}) \triangleq \mathbb{E}(t),
$$

where the matrix-valued function $\mathbb{E}$ is the third component of the admissible triplet $(\vec{u}, \vec{y}, \mathbb{E})$.

From Definitions 2 and 3 it follows that

$$
\Gamma\left(\Phi_{\text {vel }}(\tau, \cdot) \times \Phi_{\text {stress }}(\tau, \cdot)\right)=\left.\Xi\left(U_{\text {ad }}\right)\right|_{t=\tau}, \quad \forall \tau \in[0, T] .
$$

The next statement is an important consequence of Proposition 1.

Proposition 3. Under conditions (i) and (iii) from Section 3, we have

$$
\begin{gathered}
\left\|\Phi_{\mathrm{vel}}\left(t, \vec{u}_{1}\right)-\Phi_{\mathrm{vel}}\left(t, \vec{u}_{2}\right)\right\|_{L_{2}} \leq \sqrt{\frac{2 \Pi\left(\left\|\vec{u}_{1}-\vec{u}_{2}\right\|_{L_{2}}\right)}{\operatorname{Re}}}, \\
\left\|\Phi_{\text {stress }}\left(t, \vec{u}_{1}\right)-\Phi_{\text {stress }}\left(t, \vec{u}_{2}\right)\right\|_{L_{2}} \leq 2 \sqrt{\frac{a \Pi\left(\left\|\vec{u}_{1}-\vec{u}_{2}\right\|_{L_{2}}\right)}{W i}},
\end{gathered}
$$

for any $\vec{u}_{1}, \vec{u}_{2} \in U_{\mathrm{ad}}$ and $t \in[0, T]$. 
Let us consider the cost functional $\mathcal{J}_{T, \vec{b}}: U_{\mathrm{ad}} \rightarrow \mathbb{R}$ defined as follows:

$$
\mathcal{J}_{T, \vec{b}}(\vec{u}) \triangleq\left\|\Phi_{\mathrm{vel}}(T, \vec{u})-\vec{b}\right\|_{L_{2}} .
$$

Definition 4. The vector function $\vec{u}_{\star}$ from the admissible controls set $U_{\mathrm{ad}}$ is called an optimal control for integro-differential system (1)-(5) (or in other words, $\vec{u}_{\star}$ is a solution of optimization problem (6)) if

$$
\vec{u}_{\star}=\underset{\vec{u} \in U_{\mathrm{ad}}}{\arg \min } \mathcal{J}_{T, \vec{b}}(\vec{u}) .
$$

By $U_{\text {opt }}$ we denote the set of all optimal controls for (1)-(5).

Now we are ready to formulate and prove the main result of this work.

Theorem 1. Suppose conditions (i)-(iii) from Section 3 hold; then there exists at least one optimal control for integro-differential system (1)-(5) - that is, the set $U_{\mathrm{opt}}$ is not empty.

Proof. First, let us show that the admissible controls set $U_{\text {ad }}$ is compact in the space $H$.

From condition (iii) and Lemma 2, it follows that $U_{\text {ad }}$ is relatively compact in $H$.

We claim that $U_{\mathrm{ad}}$ is closed in $H$. Indeed, consider a sequence $\left\{\vec{u}_{n}\right\}_{n \in \mathbb{N}} \subset U_{\mathrm{ad}}$ such that

$$
\vec{u}_{n} \text { converges to } \vec{u}_{0} \text { strongly in } H \text { as } n \rightarrow+\infty \text {. }
$$

Since the sequence $\left\{\vec{u}_{n}\right\}_{n \in \mathbb{N}}$ is bounded in $V$, without loss of generality, we can assume that there exists a vector function $\vec{u}_{\#} \in V$ such that

$$
\vec{u}_{n} \text { converges to } \vec{u}_{\#} \text { weakly in } V \text { as } n \rightarrow+\infty \text {. }
$$

In view of (iii), the set $U_{\text {ad }}$ is convex and closed in $V$, and hence this set is weakly closed in $V$. Therefore, from (43) it follows that the inclusion $\vec{u}_{\#} \in U_{\text {ad }}$ holds. On the other hand, using Lemma 2 and (43), we deduce that

$$
\vec{u}_{n} \text { converges to } \vec{u}_{\#} \text { strongly in } H \text { as } n \rightarrow+\infty \text {. }
$$

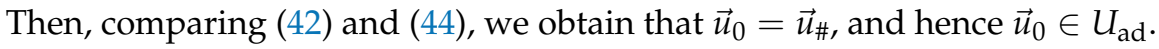

From inequality (40) it follows that the operator $\Phi_{\mathrm{vel}}(T, \cdot)$ is a continuous map from $U_{\text {ad }} \subset H$ into $H$. Consequently, the set of final states $\Phi_{\text {vel }}\left(T, U_{\text {ad }}\right)$ is compact in $H$. From the Weierstrass extreme value theorem, it follows that there exists an element $\vec{q}_{\star} \in \Phi_{\mathrm{vel}}\left(T, U_{\mathrm{ad}}\right)$ such that

$$
\left\|\vec{q}_{\star}-\vec{b}\right\|_{L_{2}}=\inf \left\{\|\vec{q}-\vec{b}\|_{L_{2}}: \vec{q} \in \Phi_{\mathrm{vel}}\left(T, U_{\mathrm{ad}}\right)\right\} .
$$

Consider a vector function $\vec{u}_{\star} \in U_{\text {ad }}$ that satisfies the equality $\Phi_{\text {vel }}\left(T, \vec{u}_{\star}\right)=\vec{q}_{\star}$ and rewrite (45) as follows:

$$
\left\|\Phi_{\mathrm{vel}}\left(T, \vec{u}_{\star}\right)-\vec{b}\right\|_{L_{2}}=\inf \left\{\|\vec{q}-\vec{b}\|_{L_{2}}: \vec{q} \in \Phi_{\mathrm{vel}}\left(T, U_{\mathrm{ad}}\right)\right\} .
$$

It is clear that equality (46) is equivalent to (41). Therefore, we deduce that $\vec{u}_{\star}$ is an optimal control in model (1)-(5), which completes the proof.

Remark 2. The solvability of an optimization problem for the Navier-Stokes equations (in our notation, the particular case $a=0$ ) with an initial control was established in [35] under the assumption that $U_{\mathrm{ad}}=V$.

Funding: This research received no external funding.

Institutional Review Board Statement: Not applicable.

Informed Consent Statement: Not applicable. 
Data Availability Statement: Not applicable.

Acknowledgments: The author thanks Evgenii S. Baranovskii for useful discussions.

Conflicts of Interest: The author declares no conflict of interest.

\section{Abbreviations}

For the reader's convenience, we collect here the main notation used in this paper.

\begin{tabular}{|c|c|}
\hline Symbols & Meaning \\
\hline$x_{1}, x_{2}$ & space variables \\
\hline$t$ & time \\
\hline$T$ & final moment of time \\
\hline $\mathcal{O}$ & flow region \\
\hline$Q_{T}$ & cylinder $\mathcal{O} \times(0, T)$ \\
\hline$\vec{y}$ & velocity field \\
\hline$\vec{b}$ & target function \\
\hline$\vec{u}$ & control function \\
\hline $\mathbb{E}$ & "elastic part" of the stress tensor \\
\hline $\mathbb{D}(\vec{y})$ & strain velocity tensor \\
\hline$\pi$ & pressure function \\
\hline $\operatorname{Re}$ & Reynolds number \\
\hline Wi & Weissenberg number \\
\hline$a$ & coupling parameter \\
\hline$U_{\mathrm{ad}}$ & admissible controls set \\
\hline$\Xi\left(U_{\mathrm{ad}}\right)$ & set of all admissible triplets \\
\hline $\mathcal{J}_{T, \vec{b}}$ & cost functional \\
\hline$C_{k}$ & positive constants that depend only on data of model (1)-(5) \\
\hline$\Gamma(\mathcal{A})$ & graph of $\mathcal{A}$ \\
\hline grad & gradient with respect to the space variables $x_{1}, x_{2}$ \\
\hline $\operatorname{div}$ & divergence with respect to the space variables $x_{1}, x_{2}$ \\
\hline$\Delta$ & Laplacian with respect to the space variables $x_{1}, x_{2}$ \\
\hline$\hookrightarrow$ & continuous dense embedding \\
\hline $\mathbb{M}_{\text {sym }}^{n \times n}$ & space of symmetric matrices of dimension $n \times n$ \\
\hline$L_{p}$ & Lebesgue space \\
\hline$W_{q}^{k}$ & Sobolev space \\
\hline $\mathcal{V}^{\mathrm{s}}$ & $\left\{\vec{\varphi} \in C^{\infty}\left(\mathcal{O}, \mathbb{R}^{2}\right): \operatorname{div} \vec{\varphi}=0\right.$ and $\left.\operatorname{supp} \vec{\varphi} \subset \mathcal{O}\right\}$ \\
\hline$H$ & closure of $\mathcal{V}$ in $L_{2}\left(\mathcal{O}, \mathbb{R}^{2}\right)$ \\
\hline$V$ & closure of $\mathcal{V}$ in $W_{2}^{1}\left(\mathcal{O}, \mathbb{R}^{2}\right)$ \\
\hline$C([0, T] ; X)$ & space of continuous functions from $[0, T]$ into $X$ \\
\hline$L_{q}(0, T ; X)$ & space of $L_{q}$-integrable functions from $[0, T]$ into $X$ \\
\hline$\vec{z}, \mathbb{F}$ & test functions \\
\hline$\vec{y}_{n}, \mathbb{E}_{n}$ & Galerkin solutions \\
\hline $\mathscr{A}, \mathscr{B}, \mathscr{C}, \mathscr{K}, \mathscr{N}$ & auxiliary operators \\
\hline$\Phi_{\mathrm{vel}}$ & velocity control operator \\
\hline$\Phi_{\text {stress }}$ & stress control operator \\
\hline
\end{tabular}

\section{References}

1. Abergel, F.; Temam, R. On some control problems in fluid mechanics. Theor. Comput. Fluid Dyn. 1990, 1, 303-325. [CrossRef]

2. Fursikov, A.V. Optimal Control of Distributed Systems. Theory and Applications; AMS: Providence, RI, USA, 2000.

3. Fursikov, A.V. Flow of a viscous incompressible fluid around a body: Boundary-value problems and minimization of the work of a fluid. J. Math. Sci. 2012, 180, 763-816. [CrossRef]

4. Glass, O. Some questions of control in fluid mechanics. In Control of Partial Differential Equations; Springer: Berlin/Heidelberg, Germany, 2012; pp. 131-206. [CrossRef]

5. Slawig, T. Distributed control for a class of non-Newtonian fluids. J. Differ. Equ. 2005, 219, 116-143. [CrossRef]

6. Guerra, T. Distributed control for shear-thinning non-Newtonian fluids. J. Math. Fluid Mech. 2012, 14, 771-789. [CrossRef]

7. Arada, N. Distributed control for multistate modified Navier-Stokes equations. ESAIM Control Optim. Calc. Var. 2013, 19, 219-238. [CrossRef] 
8. Abraham, F.; Behr, M.; Heinkenschloss, M. Shape optimization in steady blood flow: A numerical study of non-Newtonian effects. Comput. Methods Biomech. Biomed. Eng. 2005, 8, 127-137. [CrossRef] [PubMed]

9. Kuznetsov, A.V. Optimal boundary control in the initial-boundary value problem for a model of a viscoelastic medium with the full derivative. Vestn. Voronezh. Gos. Univ. Ser. Fiz. Mat. 2008, 1, 232-248. (In Russian)

10. Doubova, A.; Fernandez-Cara, E. On the control of viscoelastic Jeffreys fluids. Syst. Control Lett. 2012, 61, 573-579. [CrossRef]

11. Baranovskii, E.S.; Artemov, M.A. Existence of optimal control for a nonlinear-viscous fluid model. Int. J. Differ. Equ. 2016, 2016, 9428128. [CrossRef]

12. Baranovskii, E.S. Solvability of the stationary optimal control problem for motion equations of second grade fluids. Sib. Electron. Math. Rep. 2012, 9, 554-560.

13. Baranovskii, E.S. An optimal boundary control problem for the motion equations of polymer solutions. Sib. Adv. Math. 2014, 24, 159-168. [CrossRef]

14. Artemov, M.A. Optimal boundary control for the incompressible viscoelastic fluid system. ARPN J. Eng. Appl. Sci. 2016, 11, 2923-2927.

15. Manakova, N.A. The optimal control problem for the model of dynamics of weakly viscoelastic fluid. Vestn. Yuzhno-Ural'skogo Gos. Univ. Seriya Mekhanika Mekh. Fiz. 2015, 7, 22-29. (In Russian)

16. Anh, C.T.; Nguyet, T.M. Optimal control of the instationary three dimensional Navier-Stokes-Voigt equations. Numer. Funct. Anal. Optim. 2016, 37, 415-439. [CrossRef]

17. Ngan, N.T.; Toi, V.M. Feedback control of Navier-Stokes-Voigt equations by finite determining parameters. Acta Math. Vietnam. 2020, 45, 917-930. [CrossRef]

18. Baranovskii, E.S. Optimal boundary control of nonlinear-viscous fluid flows. Sb. Math. 2020, 211, 505-520. [CrossRef]

19. Boldrini, J.L.; Mallea-Zepeda, E.; Rojas-Medar, M.A. Optimal boundary control for the stationary Boussinesq equations with variable density. Commun. Contemp. Math. 2020, 22, 1950031. [CrossRef]

20. Evcin, C.; Ŭgur, Ö.; Tezer-Sezgin, M. Controlling the power-law fluid flow and heat transfer under the external magnetic field using the flow index and the Hartmann number. Int. J. Comput. Methods 2020, 17, 1850143. [CrossRef]

21. Baranovskii, E.S. Optimal boundary control of the Boussinesq approximation for polymeric fluids. J. Optim. Theory Appl. 2021, 189, 623-645. [CrossRef]

22. Dong, X.; Liu, X. Multi-objective optimization of heat transfer in microchannel for non-Newtonian fluid. Chem. Eng. J. 2021, 412, 128594. [CrossRef]

23. Jeffreys, H. The Earth, 2nd ed.; Cambridge University Press: Cambridge, UK, 1929.

24. Oldroyd, J.G. On the formation of rheological equations of state. Proc. R. Soc. Lond. Ser. A 1950, 200, 523-541. [CrossRef]

25. Oldroyd, J.G. Non-Newtonian effects in steady motion of some idealized elastico-viscous liquids. Proc. R. Soc. Lond. Ser. A 1958, 245, 278-297. [CrossRef]

26. Oskolkov, A.P.; Shadiev, R. Towards a theory of global solvability on $[0, \infty)$ of initial-boundary value problems for the equations of motion of Oldroyd and Kelvin-Voight fluids. J. Math. Sci. 1994, 68, 240-253. [CrossRef]

27. Oskolkov, A.P. Smooth global solutions of initial boundary-value problems for the equations of Oldroyd fluids and of their $\varepsilon$-approximations. J. Math. Sci. 1998, 89, 1750-1763. [CrossRef]

28. Saut, J.-C. Lectures on the mathematical theory of viscoelastic fluids. In Lectures on the Analysis of Nonlinear Partial Differential Equations. Part 3; Lin, F., Zhang, P., Eds.; Int. Press: Somerville, MA, USA, 2013; pp. 325-393.

29. Baranovskii, E.S.; Artemov, M.A. Global existence results for Oldroyd fluids with wall slip. Acta Appl. Math. 2017, 147, 197-210. [CrossRef]

30. Baranovskii, E.S. Steady flows of an Oldroyd fluid with threshold slip. Commun. Pure Appl. Anal. 2019, 18, 735-750. [CrossRef]

31. Castillo, R.E.; Rafeiro, H. An Introductory Course in Lebesgue Spaces; Springer International Publishing: Cham, Switzerland, 2016. [CrossRef]

32. Agranovich, M.S. Sobolev Spaces, Their Generalizations, and Elliptic Problems in Smooth and Lipschitz Domains; Springer: Cham, Switzerland, 2015. [CrossRef]

33. Temam, R. Navier-Stokes Equations. Theory and Numerical Analysis; North-Holland: Amsterdam, The Netherlands; New York, NY, USA; Oxford, UK, 1979.

34. Lions, J.L. Quelques Methodes de Resolution des Problemes aux Limites non Lineaires; Dunod Gauthier-Villars: Paris, France, 1969.

35. Fursikov, A.V. Control problems and theorems concerning the unique solvability of a mixed boundary value problem for the three-dimensional Navier-Stokes and Euler equations. Math. USSR-Sb. 1982, 43, 251-273. [CrossRef] 